\title{
High GNG13 expression is associated with poor survival in epithelial ovarian cancer and breast cancer
}

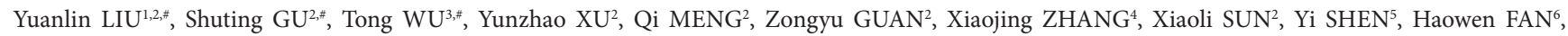 \\ Changjiang $\mathrm{GU}^{2, *}$, Jinlian FENG ${ }^{7, *}$ \\ ${ }^{1}$ Department of Obstetrics and Gynecology, Shanghai First Maternity and Infant Health Hospital, Shanghai, China; ${ }^{2}$ Department of Obstetrics \\ and Gynecology, Affiliated Hospital of Nantong University, Nantong, Jiangsu, China; ${ }^{3}$ Department of Gastroenterology, Affiliated Hospital and \\ Medical School of Nantong University, Nantong, Jiangsu, China; ${ }^{4}$ Department of Clinical Biobank, Affiliated Hospital of Nantong University, \\ Nantong, Jiangsu, China; ${ }^{5}$ Department of Epidemiology and Health Statistics, Nantong University, Nantong, Jiangsu, China; ${ }^{6}$ Department of \\ Clinical Medicine, School of Medicine, Nantong University, Nantong, Jiangsu, China; ${ }^{7}$ Department of Obstetrics and Gynecology, Nantong Ge- \\ riatric Rehabilitation Hospital, Nantong, Jiangsu, China
}

*Correspondence: cherubimliu@126.com; changjiang058@yeah.net

"Contributed equally to this work.

Received June 3, 2021 / Accepted July 15, 2021

\begin{abstract}
Recently, change in the GNG13 expression has been shown to result in multiple congenital malformations and sexual reversal, and it was also found in the brain. The aim of this study was to measure the expression levels in epithelial ovarian cancer (EOC) and breast cancer (BC) and assess their value as a potential prognostic marker. The correlation of GNG13 protein expression was detected by immunohistochemistry (IHC) in $119 \mathrm{EOC}$ and $125 \mathrm{BC}$ tissues. Assessment of the associations between GNG13 levels and various clinicopathological features was identified, the relationship between GNG13 and prognosis in BC and EOC patients was analyzed using online resources of Oncomine and Kaplan-Meier plotter. Protein expression levels of GNG13 were both significantly lower in BC and EOC compared with normal tissues ( $\mathrm{p}<0.0001$ and $\mathrm{p}<0.001$, respectively). Among the clinicopathological characteristics of $\mathrm{BC}$, tumor grade ( $\mathrm{p}=0.001)$ and TNM stage $(\mathrm{p}=0.001)$ were significantly associated with low expression of GNG13. While in EOC, low expression of GNG13 was significantly related to FIGO stage $(\mathrm{p}=0.001)$, presence of metastasis $(\mathrm{p}=0.001)$, and CA125 $(\mathrm{p}=0.001)$. Our data suggest that GNG13 expression maybe as a new inhibitor, which can strongly inhibit metastasis and partially attenuates tumor growth in $\mathrm{EOC}$ and $\mathrm{BC}$.
\end{abstract}

Key words: guanine nucleotide-binding protein 13, breast cancer, epithelial ovarian cancer, prognosis, poor survival

Understanding of breast and ovarian cancers has greatly increased over recent decades $[1,2]$. Ovarian cancer causes the greatest number of cancer deaths among women aged 50-70 years in China, and other countries [3]. In the past 30 years, $30 \%$ of patients with EOC live 5 years after their diagnoses [4]. Breast cancer (BC) has the highest morbidity and mortality of diagnosed cancers among women worldwide [5-7] but is a heterogeneous disease influenced by natural history, and environmental, genetic, behavioral, and other factors [8]. Two diseases share some risks, including diet and hormonal factors [9]. The Cancer Genome Atlas (TCGA) [7] also indicates strong gene-based similarities between BC and EOC. The discovery of BRCA1 and BRCA2 genes and their associations with $\mathrm{BC}$ and EOC $[10,11]$ prompted changes in our understanding of genetic factors in the etiology of common cancers, but only $8-10 \%$ of the diseases are caused by BRCA1/2 mutations [12, 13]. Other genome-based risk elements are likely to be identified, and their associated proteins may provide new biomarkers to diagnose and monitor BC and EOC.

Guanine nucleotide-binding protein-13 $\gamma$ (GNG13) [14], is encoded by the GNG13 gene and is mainly expressed in the ovary [15] and belongs to the guanine nucleotide-binding protein (G-protein) subunit [16]. Fujino et al. [17] found that GNG13 expression is limited to gonads during ovarian differentiation. Erickson et al. [15] found that GNG13 expression can be altered, resulting in multiple congenital malformations and sexual reversal. GNG13 has also been found in brain tissue, taste receptor cells [16], olfactory epithelium and retinal $\mathrm{ON}$ bipolar cells, and early-developing ovary. It 
is reportedly expressed in retinal and neuronal tissues and plays an especially critical role in taste signal transduction [18]. However, the role of GNG13 in tumor tissues has not been widely studied.

As patients with EOC or BC are typically diagnosed at advanced stages with few early warning symptoms, they tend to have poor prognoses. A reliable biomarker for earlystage disease is therefore urgently needed. We hypothesized that GNG13 expression could be a marker for EOC and BC. Our study used tissue microarray immunohistochemistry (TMA-IHC) to evaluate both diseases. We then analyzed the relationship between GNG13 expression and various clinicopathological features in EOC and BC. Our findings were supported by bioinformatics analysis, the Oncomine and Kaplan-Meier plotter databases.

\section{Patients and methods}

Analysis of GNG13 expression in epithelial ovarian cancer and breast cancer. To verify GNG13 expression in GC, we explored the expression of GNG13 mRNA in the Oncomine database (https://www.oncomine.org).

Patients and tissue samples - ovarian cancer. We collected specimens from 213 patients who underwent surgery for EOC at the Gynecology Department of the Affiliated Hospital of Nantong University. These tissues were embedded in paraffin while fixed with formalin. All patients underwent standardized surgery and/or chemotherapy for at least 6 cycles after resection. Of the specimens, 119 were ovarian carcinoma (84 serous carcinomas, 18 endometrioid tumors, and 17 other types); 77 showed stage I-II diseases and 42 showed stage III-IV diseases; 91 were histologically high-grade tumors and 28 were low-grade, based on the International Federation of Obstetrics and Gynecology (FIGO) criteria.

Patients and tissue samples - breast cancer. All the patients had undergone mastectomy and/or axillary dissection (radical or functional, based on clinical and surgical findings). None of the patients received preoperative radiotherapy or chemotherapy before surgery. Postoperative histological examination confirmed lymph node metastasis in all patients. The initial clinical data were collected simultaneously from the hospital's medical records, including tumor grade, hormone receptor (ER/PR) status, patient age, tumor size, ERB-B2 receptor tyrosine kinase 2 (HER2) expression, $\mathrm{Ki}-67$ status, triple-negative $\mathrm{BC}$ (TNBC) status (i.e., $\mathrm{PR}-$ / ER-/HER2- tumors), lymph node metastasis and TNM stage [19]. Tissues for the TMA were formalin-fixed and paraffin-embedded and had been obtained between 2005 and 2015. The TMA was made by Tissue Microarray System (Quick-Ray, UT06, UNITMA, Korea).

The study obtained the permission of the Human Research Ethics Committee of the Affiliated Hospital of Nantong University, Jiangsu, China. The study was approved by the Ethics Committee of the Affiliated Hospital of Nantong
University and all experiments were performed in accordance with approved guidelines of the Affiliated Hospital of Nantong University.

RNA extraction and qRT-PCR. RNAs from GIST, gastric and intestinal cancer tissues were prepared using TRIzol reagent (Invitrogen, Karlsruhe, Germany) in accordance with the manufacturer's instructions. Primers, RNA extraction, and qRT-PCR procedures are published. Primer sequences were as follows [20]: GNG13: forward 5'-CTGCTTTTGCTGTCTCCTCC-3' and reverse 5'-AGGCCAGTTGGTACTTGAGG-3' [21]. GAPDH: forward 5'-AGAAGG CTGGGGCTCATTTG-3' and reverse 5'-AGGGGCCATCCACAGTCTTC-3'.

Western blotting. Radioimmunoprecipitation (RIPA) lysis buffer containing PMSF was used to extract the cell sample protein, of which the experimental procedure was performed as described previously [22]. The rabbit antihuman GNG13 (Signalway antibody, \#46004, 1:200), antiCEMIP (cell migration-inducing protein, 1:1000, ABclonal, Woburn, MA, USA), p-STAT3 (Cell signaling Technology, \#9145, 1:2000), STAT3 (Santa Cruz, sc-8019, 1:1000) polyclonal antibody were used as primary antibodies. The PVDF membranes were incubated with the HRP-conjugated goat anti-rabbit IgG as the secondary antibody (Proteintech, SA00001-1, SA00001-2, 1:5000). Results were analyzed using Image $2 \mathrm{X}$ software (NIH, Bethesda, MD, USA).

Immunohistochemistry. The IHC methods were performed as previously described [22]. All tissue samples were fixed in $10 \%$ buffered formalin solution overnight and embedded in paraffin at room temperature. Paraffinembedded $(5 \mu \mathrm{m})$ sections were divided into core tissue biopsies ( $2 \mathrm{~mm}$ in diameter) to make TMA. Sections were deparaffinized and then incubated with $3 \% \mathrm{H}_{2} \mathrm{O}_{2}$, which was methanol for $15 \mathrm{~min}$ to quench endogenous peroxidase. Sections were then incubated with primary goat anti-GNG13 antibody (No. NBP1-91950, 1:200; Novus Biologicals, USA) overnight at $4{ }^{\circ} \mathrm{C}$. After washing with phosphate-buffered saline, sections were incubated with horseradish peroxidaseconjugated donkey anti-goat antibody (Abcam) for $15 \mathrm{~min}$ and then washed again.

Two investigators used an Olympus BX53 microscope (Olympus Co, Tokyo, Japan) to quantify GNG13 immunostaining, by scoring staining intensity as 0 (-, no staining), 1 (+, mild staining), $2(++$, medium staining), or $3(+++$, intense staining) and percentages of cells that stained positive.

We used the X-tile software program (The Rimm Lab at Yale University; http://www.tissuearray.org/rimmlab) to identify the optimal cut-off point for GNG13 IHC scores in terms of patients' overall survival (OS). We used the cut-off 120; 0-120 was considered low expression and 121-300 was high expression.

Statistical analysis. Statistical analyses $[23,24]$ of GNG13 expression were carried out using SPSS Statistics 20.0 (SPSS Inc., Chicago, IL, USA) and the Stata 12.0 (Stata Corp, College Station, TX, USA) software. We used $\chi^{2}$-tests for correlations 
between GNG13 and EOC clinicopathological factors. Multivariate Cox regression models were used to determine significant prognostic factors. Kaplan-Meier analysis and log-rank tests were used to evaluate OS. $\mathrm{p}<0.05$ was considered significant.

Bioinformatic analysis and KaplanMeier Plotter Curves. We used Oncomine (https://www.oncomine. org), which is a database of RNA and DNA sequencing information intentioned by TCGA, the Gene Expression Omnibus and other literature to evaluate the expression of CCR8 in EOC tissues, using the search terms: "GNG13", "Cancer vs. Normal Analysis," Breast Cancer "Ovarian Cancer" and "mRNA" to obtain the expression data for EOC. These data were provided in the Oncomine microarray database as the median center of $\log 2$. The KaplanMeier Plotter (http://kmplot.com/ analysis/) was used to identify the correlation between GNG13 expression and $\mathrm{OS}$ in patients with $\mathrm{EOC}$ or $\mathrm{BC}$.

\section{Results}

GNG13 expression in BC and EOC. Analysis of the data deposited in the Oncomine database indicated that the expression of GNG13 was significantly higher in $\mathrm{BC}$ and $\mathrm{EOC}$ than in normal tissues (Figures 1A, 1B). We next determined the levels of GNG13 mRNA in tissues in EOC and BC (Figures 2A, 2B). Western blot analysis was performed to detect GNG13 protein levels in EOC and BC (Figures 3A, 3B). Results showed that both mRNA and protein levels of GNG13 were higher in ovarian and breast cancer than in normal tissues.

GNG13 expression was greater in BC and EOC tissues. We used IHC to determine the GNG13 protein expression in the two cancers. GNG13 was positively stained in the cytoplasm of cancer cells. Typical GNG13 IHC staining patterns are presented in Figures $4 \mathrm{~A}$ and $4 \mathrm{~B}$.

Relationship between GNG13 expression and clinical parameters of BC/EOC. In EOC, high GNG13 expression was significantly related to
A

TCGA Breast 2 Statistics

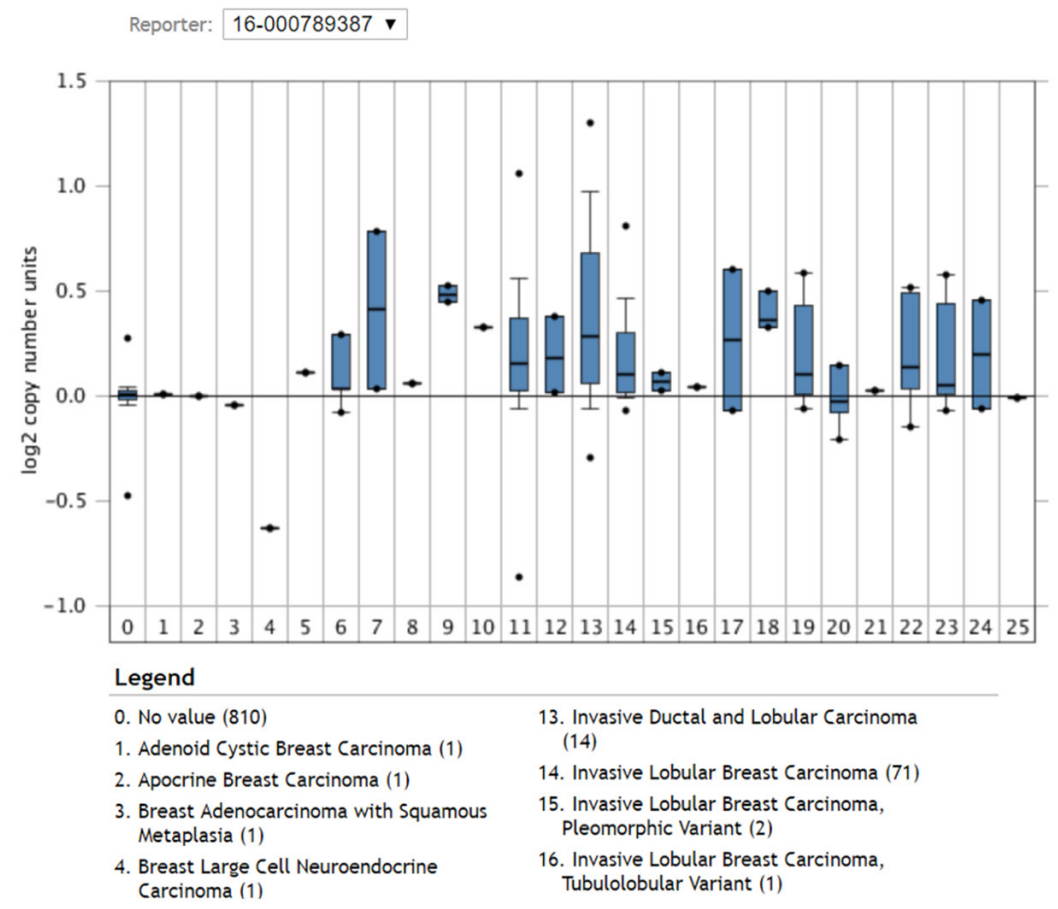

B

TCGA Ovarian Statistics

Reporter: 220806_x_at $\mathbf{v}$

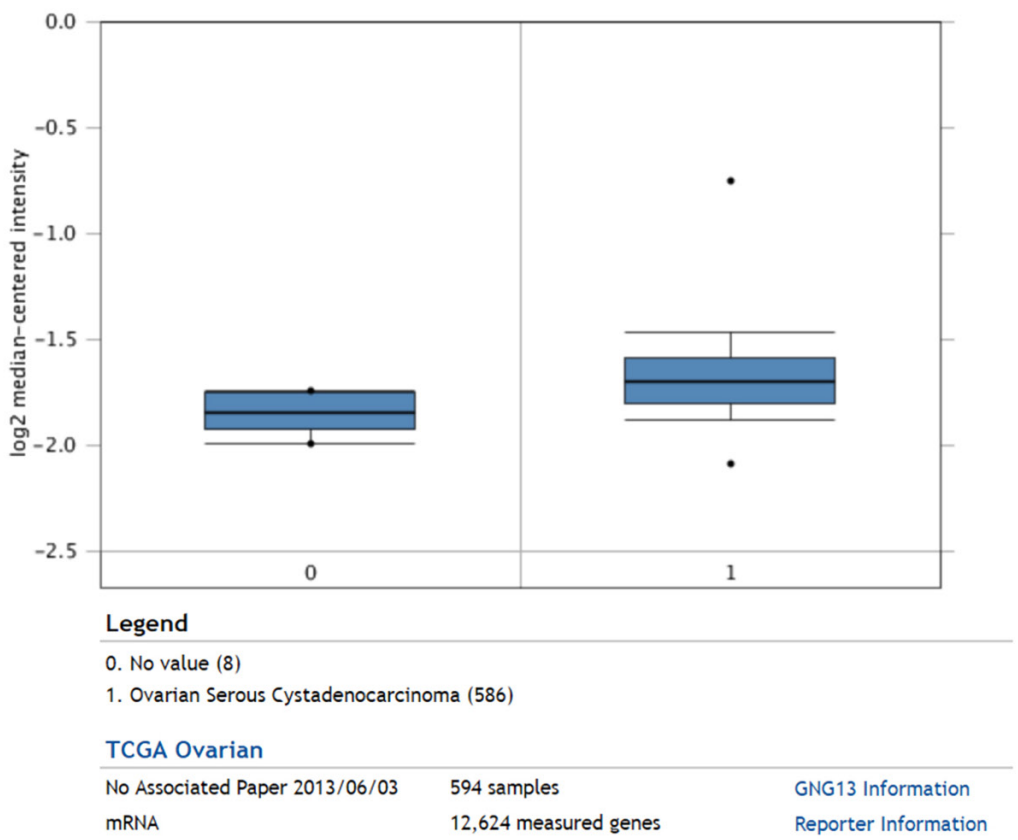

Figure 1. GNG13 mRNA is highly expressed in BC and EOC. Oncomine database showed that different expression of GNG13 was found in BC tissues and adjacent normal tissue based on the TCGA (A). Oncomine database was used to detect the expression of GNG13 in EOC by TCGA (B) 
A

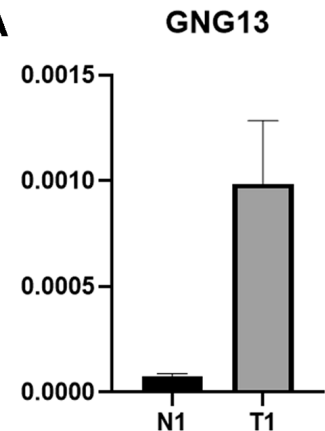

B

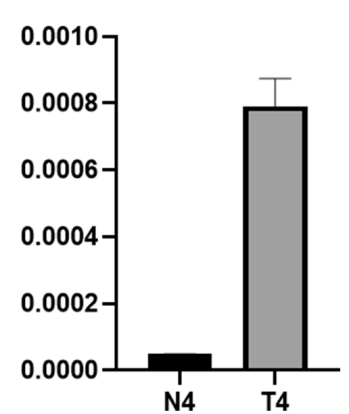

GNG13

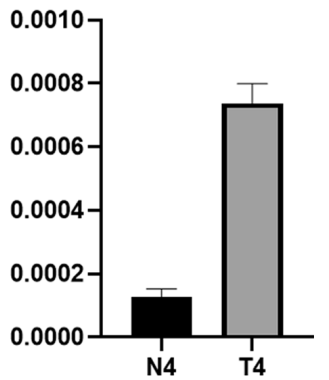

GNG13

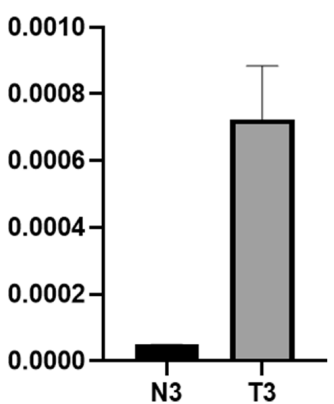

GNG13

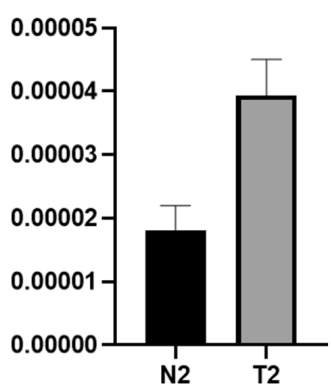

GNG13

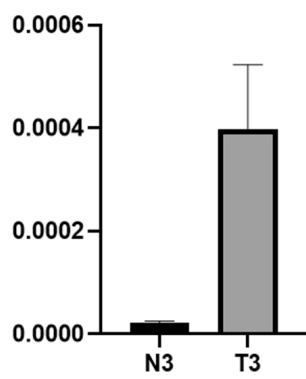

Figure 2. qRT-PCR assay was used to detect the GNG13 expression in BC (A) and EOC (B).

A

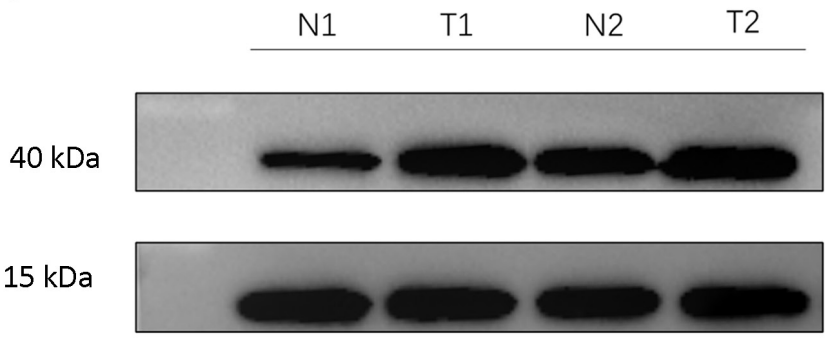

GNG13

GAPDH

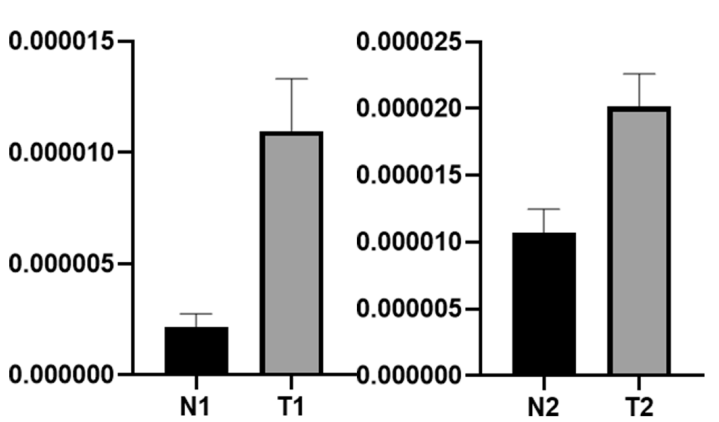

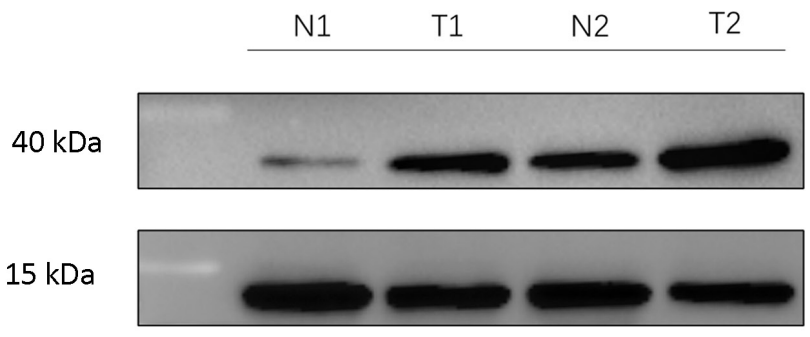

C

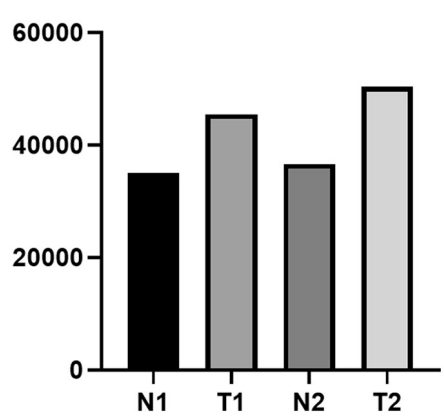

D 5000

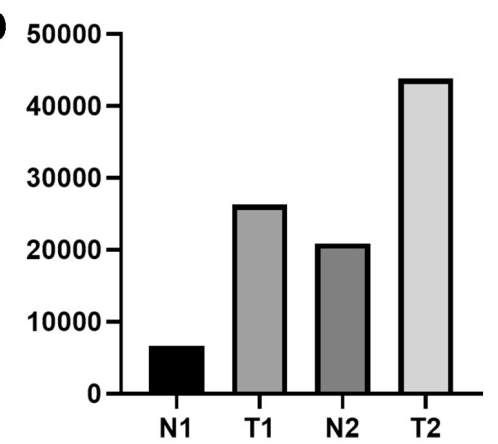

Figure 3. A) Western blot analysis of the expression of GNG13 in BC. B) Bar graph of the quantified data from A. C) Western blot analysis of the expression of GNG13 in EOC. D) Bar graph of the quantified data from C. 
Table 1. Correlation of GNG13 expression with clinicopathological characteristics in ovarian cancer.

\begin{tabular}{|c|c|c|c|c|c|}
\hline \multirow{2}{*}{ Groups } & \multicolumn{5}{|c|}{ GNG13 } \\
\hline & $\mathbf{n}$ & Low or no & High & Pearson $\chi^{2}$ & p-value \\
\hline Total & 147 & $53(36.05)$ & $94(63.94)$ & & \\
\hline Age & & & & 0.634 & 0.426 \\
\hline$\leq 60$ years & 88 & $54(61.36)$ & $34(38.64)$ & & \\
\hline$>60$ years & 59 & $40(67.80)$ & $19(36.20)$ & & \\
\hline FIGO stage & & & & 19.477 & $0.001^{*}$ \\
\hline $1-2$ & 63 & $53(84.13)$ & $10(15.87)$ & & \\
\hline $3-4$ & 84 & $41(48.81)$ & $43(51.19)$ & & \\
\hline Grade & & & & 7.609 & 0.006 \\
\hline Low grade & 32 & $14(43.75)$ & $18(56.25)$ & & \\
\hline High grade & 114 & $80(70.18)$ & $34(29.82)$ & & \\
\hline \multicolumn{3}{|c|}{ Histological classification } & & 0.573 & 0.751 \\
\hline $\begin{array}{l}\text { Serous } \\
\text { carcinoma }\end{array}$ & 111 & $73(65.77)$ & $38(34.23)$ & & \\
\hline $\begin{array}{l}\text { Endometrioid } \\
\text { carcinoma }\end{array}$ & 14 & $9(64.29)$ & $5(35.71)$ & & \\
\hline Other ${ }^{\mathrm{a}}$ & 21 & $12(57.14)$ & $9(42.86)$ & & \\
\hline Lymph nodes & & & & 2.079 & 0.149 \\
\hline Yes & 117 & $72(61.54)$ & $45(38.46)$ & & \\
\hline No & 29 & $22(75.86)$ & $7(24.14)$ & & \\
\hline Metastasis & & & & 14.722 & $0.001^{*}$ \\
\hline Yes & 83 & $42(50.60)$ & $41(49.40)$ & & \\
\hline No & 64 & $52(81.25)$ & $12(18.75)$ & & \\
\hline Single or double & & & & 2.161 & 0.142 \\
\hline Single & 94 & $56(59.57)$ & $38(40.43)$ & & \\
\hline double & 53 & $38(71.70)$ & $15(28.30)$ & & \\
\hline CA199 & & & & 4.477 & 0.107 \\
\hline Yes & 89 & $62(69.66)$ & $27(30.34)$ & & \\
\hline No & 20 & $13(65.00)$ & $7(35.00)$ & & \\
\hline Unknow & 38 & $19(50.00)$ & $19(50.00)$ & & \\
\hline CA125 & & & & 43.897 & $0.001^{*}$ \\
\hline$\leq 100$ & 29 & $4(13.79)$ & $25(86.21)$ & & \\
\hline$>100$ & 101 & $81(80.20)$ & $20(19.80)$ & & \\
\hline
\end{tabular}

Notes: ${ }^{\star} \mathrm{p}<0.05$ indicates a significant association among the variables; metastasis-pelvic lymph node metastases or nearby tissues and organs involved; aothers: clear cell carcinoma- 5 cases; mucinous carcinoma- 6 cases; transitional cell carcinoma-3 cases; adeno-squamous carcinoma-3 cases
Table 2. Correlation between the GNG13 expression and clinicopathological characteristics in breast cancer.

\begin{tabular}{|c|c|c|c|c|c|}
\hline \multirow{2}{*}{ Characteristic } & \multicolumn{5}{|c|}{ GNG13 expression (\%) } \\
\hline & $\mathbf{n}$ & Low or no & High & Pearson $\chi^{2}$ & p-value \\
\hline Age (years) & & & & 1.184 & 0.553 \\
\hline$\leq 40$ & 11 & $4(36.36)$ & $7(63.64)$ & & \\
\hline $40-60$ & 75 & $35(46.67)$ & $40(53.33)$ & & \\
\hline$\geq 60$ & 39 & $21(53.85)$ & $18(46.15)$ & & \\
\hline Tumor size $(\mathrm{cm})$ & & & & 1.854 & 0.173 \\
\hline$\leq 2 \mathrm{~cm}$ & 60 & $25(41.67)$ & $35(58.33)$ & & \\
\hline$>2 \mathrm{~cm}$ & 65 & $35(53.85)$ & $30(46.15)$ & & \\
\hline Tumor grade & & & & 71.226 & $0.001^{*}$ \\
\hline I-II & 55 & $3(5.45)$ & $52(94.55)$ & & \\
\hline III & 70 & $57(81.43)$ & $13(18.57)$ & & \\
\hline ER & & & & 0.879 & 0.348 \\
\hline Negative & 55 & $29(52.73)$ & $26(47.27)$ & & \\
\hline Positive & 70 & $31(44.29)$ & $39(55.71)$ & & \\
\hline PR & & & & 0.006 & 0.939 \\
\hline Negative & 85 & $41(48.24)$ & $44(51.76)$ & & \\
\hline Positive & 40 & $19(47.50)$ & $21(52.50)$ & & \\
\hline HER-2 expression & & & & 0.045 & 0.832 \\
\hline Negative & 99 & $48(48.48)$ & $51(51.52)$ & & \\
\hline Positive & 26 & $12(46.15)$ & $14(53.85)$ & & \\
\hline $\mathrm{Ki}-67$ & & & & 1.418 & 0.234 \\
\hline Low & 59 & $25(6.74)$ & $34(93.26)$ & & \\
\hline High & 66 & $35(53.03)$ & $31(46.97)$ & & \\
\hline $\begin{array}{l}\text { Molecular } \\
\text { classification }\end{array}$ & & & & 2.729 & 0.142 \\
\hline Luminal A & 38 & $14(36.84)$ & $24(63.16)$ & & \\
\hline Luminal B & 32 & $17(53.13)$ & $15(46.88)$ & & \\
\hline $\begin{array}{l}\text { Her2- } \\
\text { overexpression }\end{array}$ & 23 & $12(52.17)$ & $11(47.83)$ & & \\
\hline TNBC & 32 & $17(53.13)$ & $15(46.88)$ & & \\
\hline $\mathrm{N}$ stage & & & & 4.477 & 0.107 \\
\hline No & 86 & $30(34.88)$ & $56(65.12)$ & & \\
\hline $\mathrm{N} 1+2+3$ & 39 & $30(76.92)$ & $9(23.08)$ & & \\
\hline TNM stage & & & & 18.066 & $0.001^{*}$ \\
\hline Stage I-II & 58 & $16(27.59)$ & $42(72.41)$ & & \\
\hline Stage III & 67 & $44(65.67)$ & $23(34.33)$ & & \\
\hline
\end{tabular}

Note: ${ }^{*} \mathrm{p}<0.05$ indicates a significant association among the variables
FIGO stage $(\mathrm{p}=0.001)$, presence of metastasis $(\mathrm{p}=0.001)$, and CA125 ( $\mathrm{p}=0.001)$, but had no significant relationship to patients' age, tumor grade, histological classification, lymph node involvement, or CA199 expression (Table 1). In BC, high level of GNG13 expression was associated with tumor grade $(\mathrm{p}=0.001)$ and TNM stage $(\mathrm{p}=0.001)$. These data imply that EOC metastasis is related to GNG13 expression (Table 1 and Table 2).

Survival analysis. Univariate analysis showed that OS was associated with GNG13 expression $(p<0.001)$, tumor grade $(\mathrm{p}=0.031)$, CA125 expression $(\mathrm{p}<0.001)$, lymph node involvement $(\mathrm{p}=0.002)$, metastasis $(\mathrm{p}<0.001)$, subtype $(\mathrm{p}=0.029)$, and FIGO stage $(\mathrm{p}<0.001)$ among patients with
EOC; and with GNG13 expression $(\mathrm{p}<0.001)$, histological grade $(\mathrm{p}<0.001)$, and TNM stage $(\mathrm{p}<0.001)$ among patients with BC (Table 3).

In multivariate analysis, OS was independently associated with GNG13 expression (hazard ratio [HR]: 0.259; $\mathrm{p}=0.003)$, FIGO stage ([HR]: 1.853; $\mathrm{p}=0.038)$, and age ([HR]: $1.815 ; \mathrm{p}=0.013)$ among patients with EOC; and with GNG13 expression ([HR]: 0.292, $\mathrm{p}=0.014)$ and TNM stage $([\mathrm{HR}]$ : 2.664, $\mathrm{p}=0.003$ ) among patients with BC (Table 4).

Kaplan-Meier survival curves showed that both EOC patients and BC patients with low or no GNG13 expression had shorter OS than did their respective counterparts with high levels; and that patients with high FIGO stage 


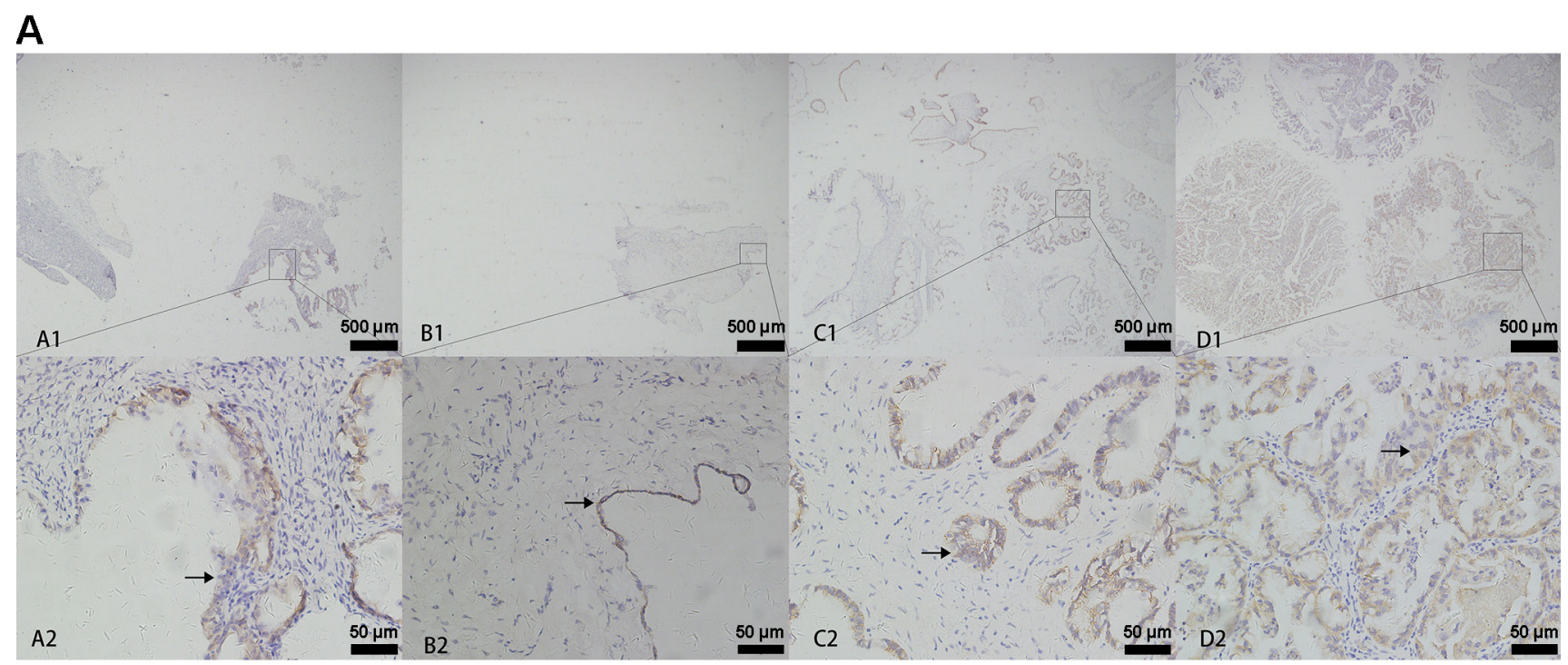

B

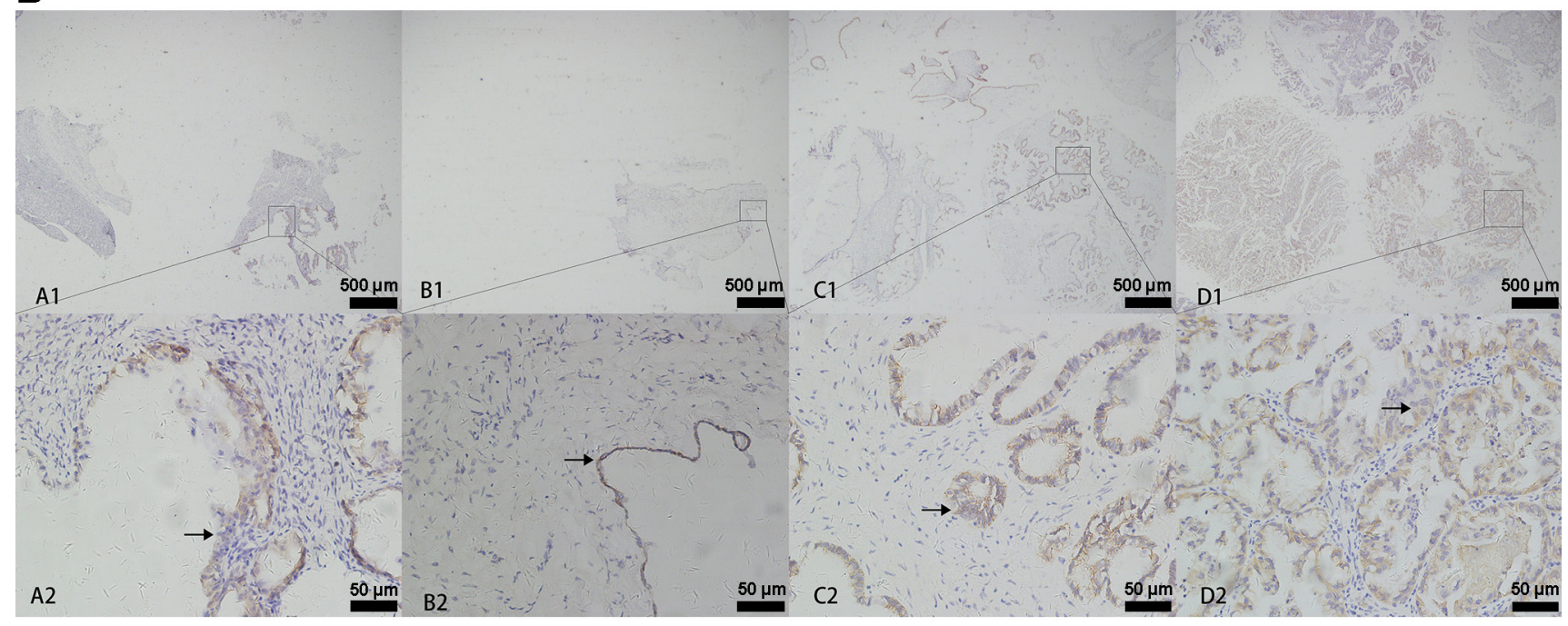

Figure 4. A) IHC analysis of GNG13 expression in BC (TMA). (A1) High IHC staining of GNG13 in the cytoplasm of the poorly differentiated invasive breast cancer cells. (B) High IHC staining of GNG13 in moderately differentiated invasive ductal carcinoma. (C) Low IHC staining of GNG13 in non-cancerous breast tissue cells. (D) Low IHC staining of GNG13 in the breast ductal papilloma cells. Original magnification 40× in (A-D); 400 $\times$ in (A1-D1). B) GNG13 protein level in EOC tissues and normal and benign ovarian tissues by IHC. (A) Weak strong IHC staining of GNG13 in benign ovarian tumor. (B) Weak IHC staining of GNG13 in normal ovarian tissue. (C) Strong IHC staining of GNG13 in poorly differentiated EOC samples. (D) Strong IHC staining of GNG13 in highly differentiated EOC samples. Original magnification 40× in (A-D); 400× in (A1-D1).

EOC (Figures 5A-5C) and patients with TNM stage III BC (respectively) had shorter OS than patients with the lowerstage disease (Figures 5D, 5E).

Association between GNG13 expression and prognosis in Oncomine and Kaplan-Meier Plotter. To verify our finding of the relationship between OS and GNG13 expression in EOC and $\mathrm{BC}$, we used Oncomine to analyze our data. The red column revealed the GNG13 mRNA upregulation. Consistent with our conclusions, GNG13 was lowly expressed in both cancers compared with normal tissues (Figure 6). The Kaplan-Meier Plotter found that high GNG13 expression is a prognostic factor for OS ([HR]: $0.84 ; 95 \%$ CI: $0.75-0.94, p=0.0017$; Figure 7).

\section{Discussion}

To our knowledge, this is the first study to use TMA-IHC and bioinformatics analysis to investigate correlations 
Table 3. Univariate and multivariate Cox proportional hazard model analysis of prognostic markers for overall survival in ovarian cancer.

\begin{tabular}{|c|c|c|c|c|c|c|}
\hline \multirow{2}{*}{ Variable } & \multicolumn{3}{|c|}{ Univariate analysis } & \multicolumn{3}{|c|}{ Multivariate analysis } \\
\hline & HR & p-value & $95 \% \mathrm{CI}$ & HR & p-value & $95 \%$ CI \\
\hline \multicolumn{7}{|l|}{ GNG13 expression } \\
\hline Low vs. High & 0.219 & $0.001^{*}$ & $0.123-0.391$ & 0.259 & $0.003^{*}$ & $0.105-0.637$ \\
\hline \multicolumn{7}{|l|}{ Age (years) } \\
\hline$\leq 60$ vs. $>60$ & 1.890 & $0.003^{*}$ & $1.236-2.890$ & 1.815 & $0.013^{*}$ & $1.134-2.903$ \\
\hline \multicolumn{7}{|l|}{ Grade } \\
\hline Low vs. high & 1.924 & $0.031^{*}$ & $1.062-3.486$ & & & \\
\hline \multicolumn{7}{|l|}{ Single or double } \\
\hline None vs. yes & 1.460 & 0.090 & $0.942-2.262$ & & & \\
\hline \multicolumn{7}{|l|}{ CA125 } \\
\hline None vs. yes & 3.747 & $0.001^{*}$ & $1.711-8.205$ & & & \\
\hline \multicolumn{7}{|l|}{ Lymph nodes } \\
\hline None vs. yes & 2.117 & $0.002^{*}$ & $1.314-3.411$ & & & \\
\hline \multicolumn{7}{|l|}{ Metastasis } \\
\hline None vs. yes & 3.969 & $0.001^{*}$ & $2.515-6.263$ & & & \\
\hline \multicolumn{7}{|l|}{ Type } \\
\hline Serous vs. others & 0.770 & $0.029^{\star}$ & $0.609-0.974$ & & & \\
\hline \multicolumn{7}{|l|}{ Ascites cell } \\
\hline None vs. yes & 1.608 & 0.153 & $0.839-3.082$ & & & \\
\hline \multicolumn{7}{|l|}{ FIGO } \\
\hline Stage I vs. stage II-IV & 2.630 & $0.001^{\star}$ & $1.788-3.867$ & 1.853 & $0.038^{*}$ & $1.034-3.319$ \\
\hline
\end{tabular}

Note: ${ }^{*} \mathrm{p}<0.05$; Abbreviations: Sc-serous carcinoma; Ec-endometrioid carcinoma; HR-Hazard ratio; CI-Confidence interval.

Table 4. Univariate and multivariate analysis of prognostic markers for overall survival in breast cancer.

\begin{tabular}{|c|c|c|c|c|c|c|}
\hline \multirow[t]{2}{*}{ Variable } & \multicolumn{3}{|c|}{ Univariate analysis } & \multicolumn{3}{|c|}{ Multivariate analysis } \\
\hline & HR & p-value & 95\% CI & HR & p-value & $95 \% \mathrm{CI}$ \\
\hline \multicolumn{7}{|l|}{ GNG13 expression } \\
\hline High vs. low & 0.161 & $0.001^{*}$ & $0.087-0.300$ & 0.292 & $0.014^{*}$ & $0.110-0.777$ \\
\hline \multicolumn{7}{|l|}{ Age (years) } \\
\hline$\leq 60$ vs. $>60$ & 1.121 & 0.602 & $0.730-1.721$ & & & \\
\hline \multicolumn{7}{|l|}{ ER expression } \\
\hline Positive vs. negative & 0.863 & 0.570 & $0.520-.1433$ & & & \\
\hline \multicolumn{7}{|l|}{ PR expression } \\
\hline Positive vs. negative & 0.583 & 0.857 & $0.493-1.488$ & & & \\
\hline \multicolumn{7}{|l|}{ Her2 expression } \\
\hline Positive vs. negative & 0.974 & 0.935 & $0.517-1.834$ & & & \\
\hline \multicolumn{7}{|l|}{$\mathrm{Ki}-67$ expression } \\
\hline Low vs. high & 1.331 & 0.276 & $0.796-2.224$ & & & \\
\hline \multicolumn{7}{|l|}{ Molecular classification } \\
\hline $\begin{array}{l}\text { Luminal A vs. luminal B } \\
\text { vs. Her-2 overexpression } \\
\text { vs. triple negative }\end{array}$ & 1.090 & 0.435 & $0.878-1.352$ & & & \\
\hline \multicolumn{7}{|l|}{ Histological grade } \\
\hline I vs. II vs. III & 4.391 & $0.001^{*}$ & $2.328-8.283$ & 1.183 & 0.730 & $0.456-3.070$ \\
\hline \multicolumn{7}{|l|}{$\mathrm{N}$ stage } \\
\hline N0 vs. $\mathrm{N} 1+2+3$ & 2.123 & 0.004 & $1.273-3.541$ & 1.331 & 0.314 & $0.763-2.319$ \\
\hline \multicolumn{7}{|l|}{ TNM stage } \\
\hline Stage I-II vs. stage III & 4.337 & $0.001^{\star}$ & $2.338-8.046$ & 2.664 & $0.004^{*}$ & $1.371-5.175$ \\
\hline
\end{tabular}

Note: ${ }^{*} \mathrm{p}<0.05$; Abbreviations: ER-estrogen receptor; PR-progesterone receptor; T-tumor stage; N-lymph node metastasis stage; TNM-tumor-node metastasis; HR-hazard ration; CI-confidence interval 

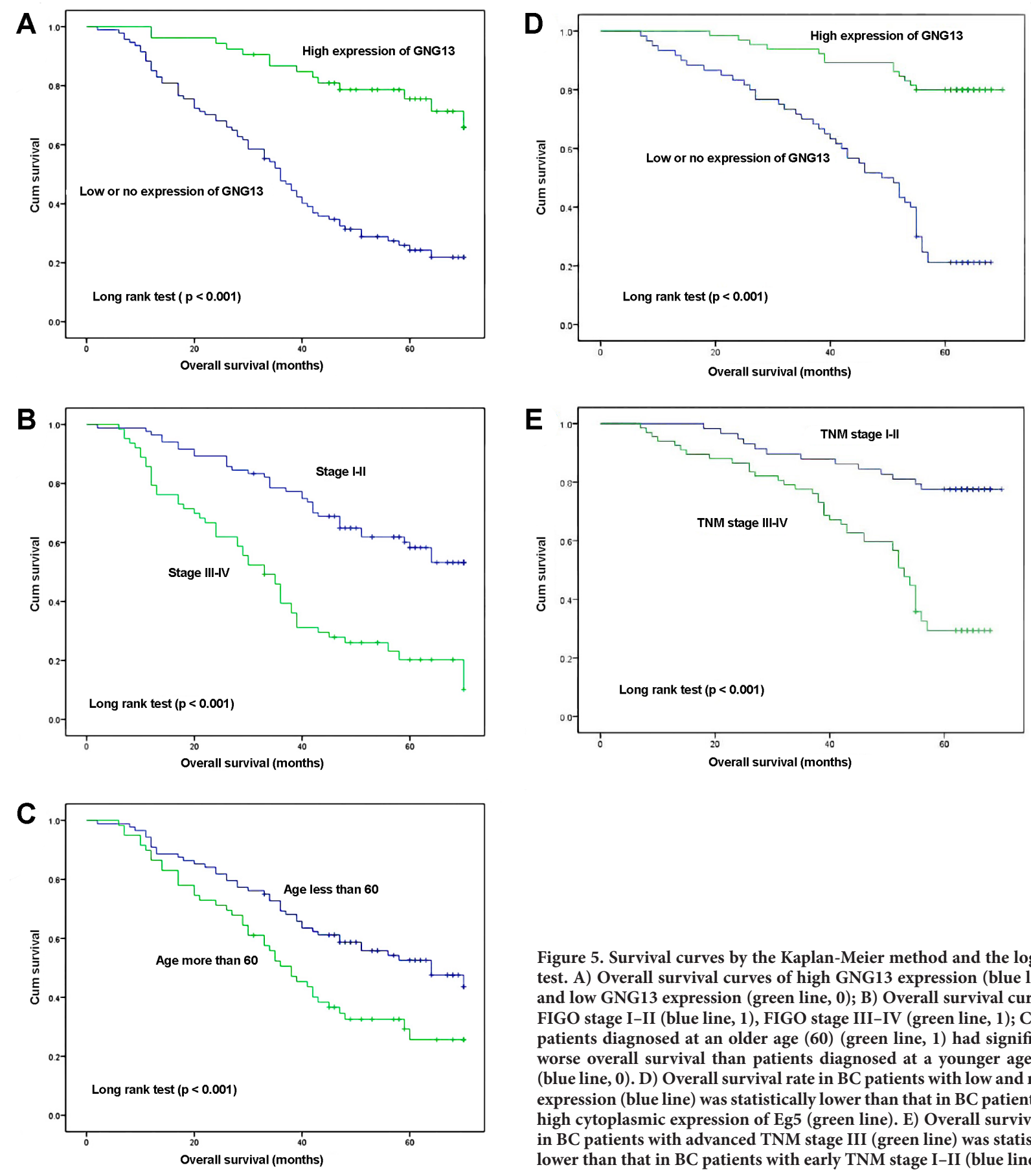

Figure 5. Survival curves by the Kaplan-Meier method and the log-rank test. A) Overall survival curves of high GNG13 expression (blue line, 1) and low GNG13 expression (green line, 0); B) Overall survival curves by FIGO stage I-II (blue line, 1), FIGO stage III-IV (green line, 1); C) EOC patients diagnosed at an older age (60) (green line, 1) had significantly worse overall survival than patients diagnosed at a younger age $(<60)$ (blue line, 0). D) Overall survival rate in BC patients with low and no Eg5 expression (blue line) was statistically lower than that in BC patients with high cytoplasmic expression of Eg5 (green line). E) Overall survival rate in BC patients with advanced TNM stage III (green line) was statistically lower than that in BC patients with early TNM stage I-II (blue line).

between GNG13 protein levels and clinicopathological features of patients with EOC and BC. We detected that GNG13 protein expression in EOC and BC tissues is significantly lower than in non-cancerous tissues, while EOC and BC patients with high expression of GNG13 suffered a poor overall survival rate. To confirm our conclusions, the bioinformatics databases were used to identify relationships

between GNG13 expression and prognosis, which were consistent with our analysis.

GNG13 is part of a G-protein family that includes Ga, $G \beta$, and $G \gamma$ subunits [14]. It couples metabolic receptors and downstream effectors. GNG13 is essential for photoreactions in all retinas in bipolar cells and is involved in ovarian development [15]. Li et al. found that abnormal expression 


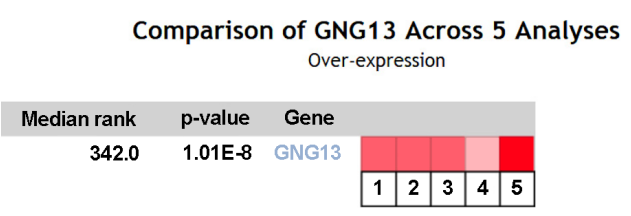

Legend

1. Chromophobe Renal Cell Carcinoma vs. Normal

Jones Renal, Clin Cancer Res, 2005

4. Renal Pelvis Urothelial Carcinoma vs. Normal

Jones Renal, Clin Cancer Res, 2005

2. Papillary Renal Cell Carcinoma vs. Normal 5. Invasive Ductal Breast Carcinoma Stroma Jones Renal, Clin Cancer Res, $2005 \quad$ vs. Normal

3. Renal Oncocytoma vs. Normal

Jones Renal, Clin Cancer Res, 2005

\section{5.}

The rank for a gene is the median rank for that gene across each of the analyses.
The p-Value for a gene is its p-Value for the median-ranked analysis.

Figure 6. Two analyses were performed in comparing the RNA expression of GNG13 between cancer and normal tissue.

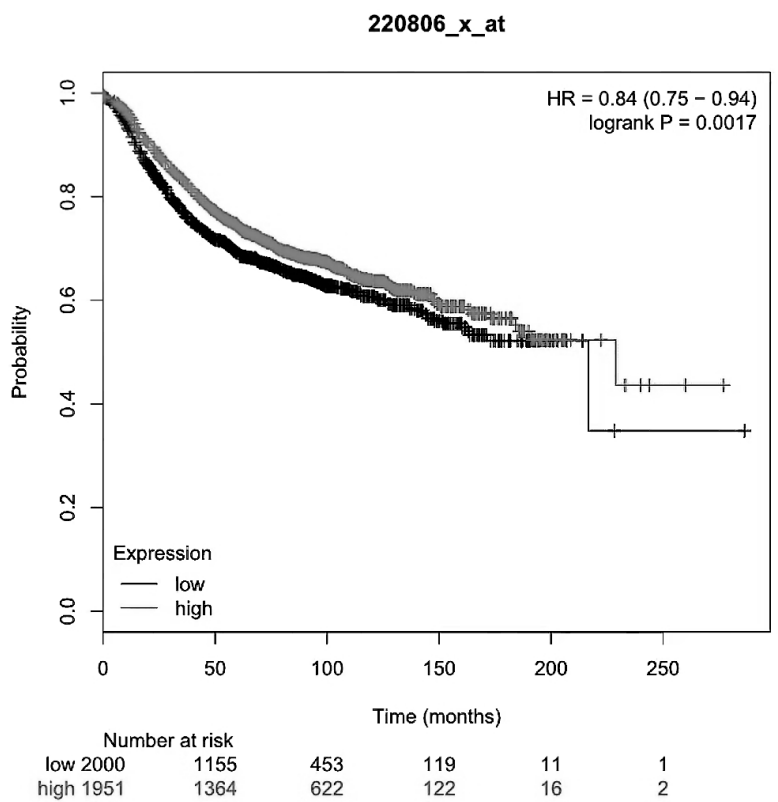

Figure 7. Prognostic value of GNG13 expression in Kaplan-Meier Plotter database. GNG13 probe number is $220806 \_\mathbf{x}$ _at. 'Probability' on the $y$-axes represents the survival rates, the red line represents the patient with GNG13 expression above the median, the black line represents the patient with GNG13 expression below the median.

of GNG13 may lead to functional constipation and proposed a new idea to understand emotional disorders [25]. These results indicate that GNG13 has a relationship with ovary development; the bioinformatics databases confirmed our prediction. However, the role of GNG13 in the development and/or progression of cancer has not been reported, as far as we know. As structures and sequence domains cannot predict function, and prior knowledge of GNG13 is limited, determining the mechanism of a metastasis-suppressing mechanism is challenging.
Knowledge of BRCA1 and BRCA2 has opened new therapeutic opportunities through a wider understanding of breast and/or ovarian tumors [26]. Our results show that high GNG13 expression in BC and EOC specimens is significantly related to better survival. This biomarker could help predict prognosis and may be a metastasis suppressor in the two diseases. Therefore, our observations increase the understanding of the role of GNG13, especially in the development and progression of $\mathrm{BC}$ and EOC.

In conclusion, our study proved that abnormal expression of GNG13 may develop migration and invasive potential of EOC and BC and it could be used as a poor prognostic factor and potential therapeutic target for EOC and BC. However, our study has some limitations. First, it is retrospectively observational, and might not represent other BC and EOC populations. Second, more work should be done to identify the function of GNG13; a genome-wide shRNA screen in vivo and in vitro study might show whether GNG13 mediates metastasis. Further prospective study of this protein's mechanisms is needed to verify our findings.

Acknowledgments: National Natural Science Foundation of China, Grant/Award Number: 81802606.

\section{References}

[1] GLOSS B S, SAMIMI G. Epigenetic biomarkers in epithelial ovarian cancer. Cancer Lett 2014; 342: 257-263. https://doi. org/10.1016/j.canlet.2011.12.036

[2] SIEGEL R, MA J, ZOU Z, JEMAL A. Cancer statistics, 2014. CA Cancer J Clin 2014; 64: 9-29. https://doi.org/10.3322/ caac. 21208

[3] LIU J, MATULONIS UA. New strategies in ovarian cancer: translating the molecular complexity of ovarian cancer into treatment advances. Clin Cancer Res 2014; 20: 5150-5156. https://doi.org/10.1158/1078-0432.CCR-14-1312

[4] WALKER JL, POWELL CB, CHEN LM, CARTER J, BAE JUMP VL et al. Society of Gynecologic Oncology recommendations for the prevention of ovarian cancer. Cancer 2015; 121: 2108-2120.

[5] XU X, TANG X, LU M, TANG Q, ZHANG H et al. Overexpression of MAGE-A9 predicts unfavorable outcome in breast cancer. Exp Mol Pathol 2014; 97: 579-584. https://doi. org/10.1016/j.yexmp.2014.11.001

[6] PARKER JS, MULLINS M, CHEANG MCU, LEUNG S, VODUC D et al. Supervised risk predictor of breast cancer based on intrinsic subtypes. J Clin Oncol 2009; 27: 11601167. https://doi.org/10.1200/JCO.2008.18.1370

[7] CANCER GENOME ATLAS NETWORK. Comprehensive molecular portraits of human breast tumours. Nature 2012; 490: 61-70. https://doi.org/10.1038/nature11412

[8] LIN H, ZHANG H, WANG J, LU M, ZHENG F et al. A novel human Fab antibody for Trop2 inhibits breast cancer growth in vitro and in vivo. Int J Cancer 2014; 134: 1239-1249. https://doi.org/10.1002/ijc.28451 
[9] HUNN J, RODRIGUEZ GC. Ovarian cancer: etiology, risk factors, and epidemiology. Clin Obstet Gynecol 2012; 55: 3-23. https://doi.org/10.1097/GRF.0b013e31824b4611

[10] BARNES DR, ANTONIOU AC. Unravelling modifiers of breast and ovarian cancer risk for BRCA1 and BRCA2 mutation carriers: update on genetic modifiers. J Intern Med 2012; 271: 331-343. https://doi.org/10.1111/j.13652796.2011.02502.x

[11] HILLER DJ, CHU QD. Current Status of Poly(ADP-ribose) Polymerase Inhibitors as Novel Therapeutic Agents for Triple-Negative Breast Cancer. Int J Breast Cancer 2012; 2012: 829315. https://doi.org/10.1155/2012/829315

[12] ZHONGQ, PENG HL, ZHAO X, ZHANG L, HWANG WT. Effects of BRCA1- and BRCA2-related mutations on ovarian and breast cancer survival: a meta-analysis. Clin Cancer Res 2015; 21: 211-220. https://doi.org/10.1158/1078-0432.CCR14-1816

[13] GANGI A, CASS I, PAIK D, BARMPARAS G, KARLAN B et al. Breast cancer following ovarian cancer in BRCA mutation carriers. JAMA Surg 2014; 149: 1306-1313. https://doi. org/10.1001/jamasurg.2014.1081

[14] KRISHNAN A, MUSTAFA A, ALMEN MS, FREDRIKSSON R, WILLIAMS MJ et al. Evolutionary hierarchy of vertebrate-like heterotrimeric $\mathrm{G}$ protein families. Mol Phylogenet Evol 2015; 91: 27-40. https://doi.org/10.1016/j.ympev.2015.05.009

[15] ERICKSON RP, YATSENKO S, LARSON K, Cheung SW. A Case of Agonadism, Skeletal Malformations, Bicuspid Aortic Valve, and Delayed Development with a 16p13.3 Duplication Including GNG13 and SOX8 Upstream Enhancers: Are Either, Both or Neither Involved in the Phenotype? Mol Syndromol 2011; 1: 185-191. https://doi.org/10.1159/000321957

[16] WALSTON ST, CHOW RH, WEILAND JD. Direct measurement of bipolar cell responses to electrical stimulation in wholemount mouse retina. J Neural Eng 2018; 15: 046003. https://doi.org/10.1088/1741-2552/aab4ed

[17] FUJINO A, PIERETTI-VANMARCKE R, WONG A, DONAHOE PK, ARANGO NA. Sexual dimorphism of G-protein subunit Gng13 expression in the cortical region of the developing mouse ovary. Dev Dyn 2007; 236: 1991-1996. https://doi.org/10.1002/dvdy.21183
[18] TUMMALA SR, NEINSTEIN A, FINA ME, DHINGRA A, VARDI N. Localization of Cacna1s to ON bipolar dendritic tips requires mGluR6-related cascade elements. Invest Ophthalmol Vis Sci 2014; 55: 1483-1492. https://doi.org/10.1167/ iovs.13-13766

[19] EDGE SB, COMPTON CC. The American Joint Committee on Cancer: the 7th edition of the AJCC cancer staging manual and the future of TNM. Ann Surg Oncol 2010; 17: 1471-1474. https://doi.org/10.1245/s10434-010-0985-4

[20] FENG Y, XUE WJ, LI P, SHA ZY, HUANG H et al. RASSF1A hypermethylation is associated with aflatoxin $\mathrm{B} 1$ and polycyclic aromatic hydrocarbon exposure in hepatocellular carcinoma. Hepatogastroenterology 2012; 59: 1883-1888. https:// doi.org/10.5754/hge11731

[21] LI F, PONISSERY-SAIDU S, YEE KK, WANG H, CHEN $\mathrm{ML}$ et al. Heterotrimeric G protein subunit Ggamma13 is critical to olfaction. J Neurosci 2013; 33: 7975-7984. https:// doi.org/10.1523/JNEUROSCI.5563-12.2013

[22] SUN R, WANG X, ZHU H, MEI H, WANG W et al. Prognostic value of LAMP3 and TP53 overexpression in benign and malignant gastrointestinal tissues. Oncotarget 2014; 5: 12398-12409. https://doi.org/10.18632/oncotarget.2643

[23] HUANG J, ZHANG J, LI H, LU Z, SHAN W et al. VCAM1 expression correlated with tumorigenesis and poor prognosis in high grade serous ovarian cancer. Am J Transl Res 2013; 5: 336-346.

[24] LI J, HUANG J, HUANG F, JIN Q, ZHU H et al. Decreased expression of IDH1-R132H correlates with poor survival in gastrointestinal cancer. Oncotarget 2016; 7: 73638-73650. https://doi.org/10.18632/oncotarget.12039

[25] LI Y, SHI L, YUE L, GAO R, YU ZQ et al. Hippocampal gene expression profiling in a rat model of functional constipation reveals abnormal expression genes associated with cognitive function. Neurosci Lett 2018; 675: 103-109. https://doi. org/10.1016/j.neulet.2018.03.023

[26] HOBERG-VETTI H, BJORVATN C, FIANE BE, AAS T, WOIE $\mathrm{K}$ et al. BRCA1/2 testing in newly diagnosed breast and ovarian cancer patients without prior genetic counselling: the DNA-BONus study. Eur J Hum Genet 2016; 24: 881-888. https://doi.org/10.1038/ejhg.2015.196 